\title{
¿Consumidores o ciudadanos? Una propuesta de inserción de la educación económica y financiera en la formación inicial docente*
}

\author{
Consumers or Citizens? An Economic and Financial Education Framework for Initial \\ Teacher Training
}

\author{
Consumidores ou cidadãos? Uma proposta de inclusão da educação financeira e \\ econômica na formação inicial de professores
}

\author{
Marianela Denegri C., ${ }^{a}$ Carlos Del Valle R., ${ }^{b}$ Yéssica González G., ${ }^{c}$ Soledad Etchebar- \\ ne L., ${ }^{d}$ Jocelyne Sepúlveda A., ${ }^{e}$ Diego Sandoval G. ${ }^{f}$ \\ a'Departamento de Psicología, Universidad de La Frontera. Av. Francisco Salazar 01145, Fono: 045 - 325605. \\ Correo electrónico: marianela.denegri@ufrontera.cl (correspondencia) \\ ${ }^{\text {b}}$ Departamento de Lenguas, Literatura y Comunicación, Universidad de La Frontera. Correo electrónico: \\ carlos.delvalle@ufrontera.cl \\ 'Departamento de Ciencias Sociales, Universidad de La Frontera. Correo electrónico: \\ yessica.gonzalez@ufrontera.cl \\ d Departamento de Administración, Universidad de Chile. Correo electrónico: setchebarne@unegocios.cl \\ eDepartamento de Psicología, Universidad de La Frontera. Correo electrónico: Correo electrónico: jocelyne. \\ sepulveda@ufrontera.cl \\ fDepartamento de Psicología, Universidad de La Frontera. Correo electrónico: dsgatica@gmail.com (tesista)
}

\section{RESUMEN}

El modelo económico chileno ha aumentado el riesgo de las operaciones financieras en una población con bajos niveles de alfabetización económica. Especialmente preocupante es la situación de profesores y estudiantes universitarios de pedagogía, por el impacto multiplicativo de su conducta en futuros estudiantes. Los objetivos fueron detectar brechas de conocimiento en educación económica y financiera, a partir de la investigación desarrollada en Chile y proponer un modelo educativo para la alfabetización económica y financiera en la formación inicial docente. Los resultados indican la precaria formación económica en el currículum educativo y la necesidad de intervenir en distintos segmentos de la población, especialmente en profesores y estudiantes de pedagogía. Se propone un modelo que integra los componentes de la educación económica y sus conceptos articuladores con los lineamientos de la OCDE, los contenidos específicos y la metodología didáctica en una propuesta de educación económica para la formación inicial docente.

Palabras clave: educación económica, educación financiera, formación inicial docente.

\begin{abstract}
The Chilean economic model has increased the risk of financial transactions in a population with low levels of economic literacy. Especially worrying is the situation of teachers and university students of pedagogy by multiplicative impact of their conduct on future students. The objectives were to detect gaps of knowledge in economic and financial education based on the review of the research developed in Chile; and to propose an educational model for financial and economic literacy in initial teacher training. The results show the precarious economic training in the educational curriculum and the need to intervene in different segments of population, especially teachers and students of pedagogy. The researchers propose a model that integrates the components of economic education and their articulators concepts with the guidelines of the OECD, the specific contents and teaching methodology in a proposal of economic education for initial teacher training.
\end{abstract}

Key words: economic education, financial education, initial teacher training.

Este trabajo se ha desarrollado y ha sido financiado por el Fondo Nacional de Ciencia y Tecnología(FONDECYT) a través del proyecto $\mathrm{N}^{\circ} 1110711$. 


\section{RESUMO}

O modelo econômico chileno tem aumentado o risco das operações financeiras para a população com baixos níveis de alfabetização econômica. Particularmente, é preocupante a situação dos professores e estudantes de Pedagogia pelo impacto das suas condutas em seus futuros estudantes. Objetivou-se detectar as lacunas de conhecimento em educação econômica e financeira, a partir de investigação desenvolvida no Chile; também propor um modelo educacional para a alfabetização econômica e financeira na formação inicial de professores. Resultados indicam a precária formação econômica no currículo educacional e a necessidade de intervir junto aos diferentes segmentos da população, especialmente, aos professores e estudantes de Pedagogia. Propõe-se um modelo que integra os componentes da educação econômica e seus conteúdos articuladores com os alinhamentos da OCDE e os conteúdos específicos bem como a metodologia de ensino em uma proposta de educação econômica para a formação inicial de professores.

Palavras chave: educação econômica, educação financeira, formação inicial de professores.

\section{INTRODUCCIÓN}

La progresiva globalización ha transformado las dinámicas relacionales al interior de la sociedad chilena y le ha otorgado un poder inusitado a ciertas prácticas, dentro de las cuales destaca de manera particular el consumo y su valoración, no sólo como un medio de satisfacción de aquellas necesidades esenciales o básicas, sino que también como un referente de definición identitaria, tanto a nivel individual como colectivo (Bauman, 2007; Denegri y Martínez, 2004).

Como señala el informe de Desarrollo Humano en Chile, correspondiente al año 2002, para muchos chilenos el consumo tiene un significado similar al que antes tenía el trabajo y con ello constituye la cristalización física de la identidad individual, al mismo tiempo que un anclaje material al vínculo social (Programa de las Naciones Unidas para el Desarrollo [PNUD], 2002). A su vez, se han producido profundos cambios en los valores de la sociedad, la que ha transitado desde una concepción que evalúa positivamente la austeridad hacia una mayor búsqueda del hedonismo y la satisfacción inmediata de los deseos, flexibilizando con ello su actitud hacia la deuda. Esto ha permitido un mayor desarrollo económico (mayor consumo), pero al mismo tiempo ha aumentado el riesgo de las operaciones financieras especialmente en las poblaciones más vulnerables como son los sectores de menores ingresos y los jóvenes (Denegri, Iturra, Palavecinos y Ripoll, 1999; McElprang, Haskell \& Jenkins, 2005; Norvilitis, Sazblicki \& Wilson, 2003; Norvilitis \& Santa María, 2002; PNUD/INJUV, 2003). De esta forma, la capacidad de manejar adecuadamente las finanzas y comprender el funcionamiento de la economía, así como de desarrollar actitudes y hábitos de consumo responsables, constituyen competencias esenciales para todos los miembros de la sociedad, incluyendo los estudiantes universitarios, y, específicamente, los estudiantes de pedagogía, por el futuro impacto, tanto en sus prácticas docentes, como por el efecto de modelaje que tiene su comportamiento en los propios educandos (Walstad \& Allgood, 1999).

En este contexto, la Alfabetización Económica debe proporcionar a las personas las herramientas para entender su mundo económico, interpretar los eventos que pueden afectarlos directa o indirectamente, y mejorar las competencias para tomar decisiones personales y sociales sobre la multitud de problemas económicos que se encuentran en la vida cotidiana (Denegri y Martínez, 2004; Yamane, 1997). Por otra parte, una adecuada Alfabetización Financiera desarrolla la habilidad para balancear ingresos y gastos, mantener una cuenta corriente saludable, preparar y administrar un presupuesto, desarrollar estrategias de ahorro y manejar en forma eficiente el uso del crédito, tanto cotidianamente, como en una proyección hacia el futuro (Commonwealth Bank Foundation [CBF], 
2004a; Denegri, Martínez y Etchebarne, 2007). Es así como la alfabetización económica y financiera es una base esencial para enfrentar y resolver los problemas económicos y financieros cotidianos, siendo vital para el logro de una vida más próspera, saludable y feliz (CBF, 2004b; National Council on Economic Education [NCEE], 2005; Santomero, 2003; Raven, 2005). Al respecto, varios estudios muestran que los problemas económicos y financieros están a la base de muchas de las dificultades que afectan la calidad de vida de los sujetos, como, por ejemplo, rupturas familiares, problemas de salud mental y otras experiencias frustrantes o insatisfactorias, al incrementar el aislamiento, el stress, la depresión y los problemas de autoestima (Lyons, 2004).

Desde la política pública, la visualización de la educación como uno de los medios más efectivos para promover la equidad en el acceso a oportunidades de mejor calidad de vida para la población hace que la atención y tensión se centre hoy en la efectividad de los procesos de formación inicial de profesores, por su impacto probable en el mejoramiento de la calidad de las competencias complejas que debe desarrollar la población escolar para responder a las nuevas necesidades de adaptación y ejercer adecuadamente su ciudadanía, aspecto esencial para el fortalecimiento de la democracia en las economías emergentes. Vinculado a ello, el Informe de la Comisión de Formación Ciudadana señala:

La formación ciudadana debe entregar herramientas fundamentales de análisis económico que permitan manejar adecuadamente los desafíos que impone la realidad. Es decir, es menester efectuar una alfabetización económica, de modo que los ciudadanos manejen adecuadamente conceptos como escasez, intercambio, costos, beneficios, precios, oferta, demanda, rentabilidad, asignación de recursos, ahorro, inversión, crecimiento, desempleo, valorización, trabajo, producción, etc. Deben destacarse las consecuencias para las personas que tiene el tema de la previsión y su adecuada comprensión (Comisión de Formación Ciudadana, 2004: 47).

Este mismo informe señala que los contenidos específicos de economía son trabajados por sólo el $18.6 \%$ de los profesores de educación básica encuestados, mientras que en educación media los porcentajes variaban entre el $83 \%$ al $49 \%$, dependiendo de la complejidad de los conceptos económicos abordados, lo que podría a su vez estar vinculado con el propio nivel de alfabetización económica y de comprensión general de la economía de los propios profesores encargados de dicha formación.

A partir de esta contextualización inicial, el presente artículo buscó profundizar en la reflexión sobre la necesidad de incorporar la educación económica como una temática importante en la formación inicial de profesores, especialmente en aquellos que por su sector de desempeño serán los responsables de la formación ciudadana y económica. A partir de ello, este trabajo tuvo como objetivos:

1. Detectar brechas de conocimiento en educación económica y financiera a partir de la revisión del estado del arte de la investigación desarrollada en Chile.

2. Proponer un modelo educativo general que estructure las acciones educativas en alfabetización económica y financiera en la formación inicial docente.

\section{BRECHAS EN EDUCACIÓN ECONÓMICA Y FINANCIERA EN CHILE}

De la revisión de la literatura sobre investigaciones en alfabetización económica y financiera en distintos segmentos de la población chilena, se identifican como variables 
relevantes edad, nivel educativo, género y nivel socioeconómico. Estas variables serán abordadas en detalle a continuación.

\subsection{ALFABETIZACIÓN ECONÓMICA EN ADULTOS DE DIFERENTE NIVEL SOCIOECONÓMICO}

En los estudios realizados con población adulta, se observa que las personas suelen dominar ciertas habilidades rudimentarias en el manejo de las finanzas personales, aunque estas no son suficientes para una adecuada comprensión del mundo económico. Dentro de las principales carencias se identifica la dificultad para comprender la naturaleza sistémica de la economía, la baja comprensión de los mecanismos de crédito y endeudamiento y los costos asociados, y la dificultad para analizar distintas alternativas de ahorro e inversión que sean rentables a corto y largo plazo (Herrera, Estrada y Denegri, 2011).

En cuanto a comportamientos de consumo, se observa una clara tendencia hacia el consumo poco reflexivo con resultados poco eficientes, dado que por lo general se gasta más de lo que se debería, lo cual aumenta la percepción de vivir en una situación financiera difícil. Estos estilos de consumo, si bien muestran una presencia transversal en los distintos estratos económicos, son más fuertes y permanentes en los segmentos socioeconómicos medios (Denegri, Gempp, Del Valle, Etchebarne y González, 2006b).

En otros estudios, se ha evidenciado la precaria alfabetización económica en jóvenes y adultos, lo que se hace más evidente en los sectores de menores ingresos y en las mujeres (Denegri, Martínez y Etchebarne, 2007; Denegri y Palavecinos, 2003; Gempp, Denegri, Caripán y Catalán, Hermosilla y Caprile, 2007). Se aprecia una comprensión básica del carácter fiduciario del dinero y de la idea de ganancia como elemento central en la economía, pero se observan dificultades en la comprensión de las relaciones complejas entre emisión y circulación monetaria y los procesos productivos. Junto con lo anterior, se concibe al Estado de un modo paternalista, adscribiéndole la potestad de regular y controlar todo el sistema económico, aspecto muy lejano de sus funciones reales en un sistema de libre mercado como el chileno (Denegri et al., 2006b).

En relación al impacto del nivel educacional, los estudios muestran que es necesario no sólo contar con herramientas cognitivas para su comprensión, sino también disponer de información específica que permita un mejor desempeño. Los resultados de las investigaciones con adultos chilenos muestran que sólo personas con un elevado nivel educacional y con trabajos que exigen un claro manejo del tema financiero alcanzan mayores niveles de alfabetización económica, en oposición a quienes presentan menores niveles de alfabetización económica, mayormente representados en los niveles educacionales más bajos y en las ocupaciones más precarias y vinculadas al comercio o los servicios (Denegri y Palavecinos, 2003). No obstante ello, no basta con la entrega de conceptos o contenidos si ello no va acompañado de la reflexión profunda sobre valores y actitudes que median en las decisiones económicas y el desarrollo de hábitos y estrategias de toma de decisiones eficientes frente a las distintas alternativas de consumo.

En esta misma línea, se aprecian diferencias evidentes asociadas al nivel socioeconómico que indican que adultos de estrato socioeconómico bajo presentan un nivel más bajo de comprensión de los conceptos y prácticas económicas necesarias para un desempeño económico eficiente, respecto a los adultos de estrato socioeconómico medio bajo, medio alto y alto. Así, en el Test de Alfabetización Económica, de un puntaje total de 27 puntos, los adultos de NSE Bajo presentan una media de 11,80 puntos; el estrato medio bajo una 
media de 17,53 puntos; el segmento medio alto una media de 17,87 puntos, mientras que el estrato socioeconómico alto presenta una media de 20,48, siendo este grupo en donde además se presenta una menor desviación típica por lo que sus conocimientos económicos tienden a ser más uniformes (Riquelme y Denegri, 2008).

\subsection{ALFABETIZACIÓN ECONÓMICA Y GÉNERO}

Una parte importante de los estudios realizados concuerdan en que existen diferencias de género tanto en los niveles de alfabetización económica como en los significados que hombres y mujeres atribuyen al dinero. Estas diferencias reproducen la adscripción de roles tradicionales de la sociedad patriarcal, apreciándose que los hombres tienden a manejarse mejor en las esferas públicas del uso del dinero, como es el acceso al crédito y la toma de decisiones de endeudamiento a largo plazo, mientras que las mujeres tienden a hacerlo en el mundo de lo privado, referido al cuidado de la casa y de la familia, es decir, organización de las compras domésticas, jerarquización de las necesidades y toma de decisiones de endeudamiento menor (Denegri, Palavecinos, Ripoll y Yáñez, 1999, Cit. en Denegri et al., 2006b; Denegri y Palavecinos, 2003).

Es probable que esta especialización por género interfiera en la posibilidad que las mujeres se aproximen con fluidez a los procesos productivos y de gestión, limitando sus oportunidades de adquirir una alfabetización económica más eficiente, y por ende, no comprendiendo los procesos económicos en su complejidad sistémica (Denegri y Palavecinos, 2003).

Por otra parte, en estudios realizados con estudiantes universitarios, emergen otras diferencias de género vinculadas a disposiciones actitudinales frente a la compra y al consumo. En un estudio finalizado el año 2011 con 1229 estudiantes de carreras pedagógicas a lo largo del país, se observan dos estilos actitudinales ante la compra que se distribuyen de manera diferencial por género. En el caso de los hombres, se observa la presencia de un estilo actitudinal con mayores tendencias hacia la racionalidad, con baja impulsividad y baja compulsividad. En cambio, en el caso de las mujeres, se aprecia un estilo actitudinal ambivalente, donde coexiste una alta racionalidad con una alta impulsividad y una compulsividad media. Este estilo corresponde claramente a un perfil de riesgo dada su dependencia del contexto y la influencia de, por ejemplo, los medios de comunicación (Denegri, Del Valle, Etchebarne y González, 2011).

\subsection{SOCIALIZACIÓN ECONÓMICA EN LA FAMILIA}

La socialización económica es definida como un proceso de aprendizaje de las pautas de interacción con el mundo económico mediante la interiorización de conocimientos, destrezas, estrategias, patrones de comportamiento y actitudes acerca del uso del dinero y su valor en la sociedad. En este proceso intervienen distintos agentes de socialización, siendo la familia el primero de ellos y probablemente uno de los más importantes (Denegri y Martínez, 2004).

En estudios sobre estrategias y prácticas de socialización económica utilizadas por familias urbanas de diversos niveles socioeconómicos, se encontró que en el discurso de padres de clase media se reconoce la importancia de educar en el uso del dinero. Sin embargo, esto no parece reflejarse coherentemente en las prácticas cotidianas de socialización 
económica, ya que si bien los padres dicen valorar un estilo de vida austero, con sus hijos se comportan más bien como consumidores impulsivos, donde la reflexión y los límites a las peticiones de consumo de los niños parecen ser bastante difusos. Por otro lado, si bien valoran la importancia de aprender a administrar el dinero, no fomentan la autonomía ni la responsabilidad efectiva de dicha administración en sus hijos. Otro resultado interesante es la tendencia a repetir, en la socialización de sus hijos, las pautas que ellos vivieron en su infancia, apreciándose la presencia de una transmisión intergeneracional de pautas económicas que no siempre es concordante con los cambios del sistema económico chileno y la consideración de los niños como un segmento de consumidores creciente (Denegri, Gempp y Martínez, 2005a; Denegri, Palavecinos, Gempp y Caprile, 2005b).

En términos de prácticas de ahorro del dinero en niños y niñas preadolescentes, se ha evidenciado la presencia de diferencias según nivel socio-económico, donde niños de NSE alto presentan una mayor tendencia al ahorro sistemático, una mejor comprensión de conceptos económicos y suelen desarrollar emprendimientos como una forma de autoabastecerse de recursos económicos; se aprecia además una alta sintonía entre las estrategias que mencionan los niños y aquello que reportan los padres como formas de socialización económica, donde destacan las prácticas de ahorro y la entrega de mesada, traduciéndose en un estilo de socialización orientado a la producción y al ahorro. Por otra parte, los niños de NSE medio presentan una baja tendencia al ahorro en general, y un uso ineficiente de la alcancía, la cual es utilizada casi como un monedero y no como una estrategia de ahorro; se aprecia además que manejan dinero de manera permanente porque sus padres se los proveen cada vez que lo piden, no observándose pautas sistemáticas como la mesada que les ayude a desarrollar estrategias de administración del dinero. Es importante en este segmento, tal como lo habíamos señalado, la dificultad que tienen los padres para desarrollar estrategias articuladas de socialización económica, observándose que tienden a satisfacer todo lo que piden los niños y a mantener, a su vez, como familia, comportamientos económicos menos reflexivos. Ello configura un estilo de socialización orientado hacia el consumismo. Finalmente, entre los niños preadolescentes de NSE bajo existe una tendencia hacia el ahorro como estrategia de afrontamiento, en el cual el dinero ahorrado se guarda en la casa o a cargo de sus padres, de modo de estar disponible para ser utilizado por la familia ante alguna eventualidad de tipo económico (Denegri, Lara, Córdoba y Del Valle, 2008).

De esta forma, la socialización económica familiar se relaciona con una probable transmisión intergeneracional de pautas de gestión del dinero, donde los niños de NSE alto reproducen las prácticas de generación y acumulación de capital que ven en sus propias familias y los niños de NSE bajo, los patrones de dependencia y subordinación laboral presentes en sus familias (Denegri et al., 2008). Los padres de NSE medio, pese a valorar un estilo de vida austero, con sus hijos se comportan como consumidores impulsivos, mostrándose difusos tanto en la reflexión como en los límites a las peticiones de consumo de los niños. Además, si bien reconocen la importancia de aprender a administrar el dinero, no suelen fomentar la autonomía ni la responsabilidad efectiva de dicha administración. Prueba de ello es el escaso uso de la mesada como mecanismo para introducir prácticas de racionalización de recursos económicos (Denegri, et al., 2005a; Denegri, del Valle, Gempp y Lara, 2006a).

\subsection{ALFABETIZACIÓN ECONÓMICA Y FINANCIERA EN LA INFANCIA}


La alfabetización económica y financiera debe estar presente desde muy temprano en la vida de los niños, porque la reflexión sistemática sobre problemas económicos reales de la vida cotidiana incrementa las capacidades de razonamiento general y ayuda a los niños a diferenciar aspectos que se consideran claves para comprender el mundo social. Uno de ellos es la comprensión de la diferencia entre una concepción pasiva de la vida, donde los individuos no tienen la posibilidad de control versus la conceptualización de los fenómenos sociales como resultado de elecciones realizadas por individuos y colectivos. El segundo aspecto clave implica la toma de conciencia que en cada decisión hay costos y beneficios y que ellos deben ser evaluados antes de actuar. Ambos aspectos requieren del desarrollo de la capacidad de relacionar procesos, evaluar cursos de acción y reflexionar sobre consecuencias, todo lo cual forma parte importante de las competencias cognitivas, conductuales y valóricas necesarias para la instalación de una lógica ciudadana en contraposición con una postura pasiva frente a la realidad social (Denegri y Martínez, 2004).

En general, las investigaciones indican que la educación económica y financiera debe iniciarse tempranamente, siendo muy importante la consideración del nivel de desarrollo cognitivo y social de los niños y sus propias concepciones en torno al mundo del dinero, la economía y el consumo.

En los pequeños, incluso desde el nivel pre-escolar, pueden integrarse actividades y experiencias con la compra; reconocimiento de monedas y billetes y, especialmente, en el desarrollo de actitudes proclives a un consumo responsable y sostenible.

Es a partir del segundo ciclo educativo donde se observa que los estudiantes de $5^{\circ}$ y $6^{\circ}$ curso son los que más se benefician de una educación económica más estructurada que integre conceptos, reflexión y aplicación a la resolución de problemas. Ello, considerando que por su etapa evolutiva han desarrollado las estructuras cognitivas operacionales necesarias para relacionar eventos y procesos, poseen las bases del pensamiento matemático y una capacidad de memoria más flexible, que les permite establecer relaciones entre experiencias pasadas y nueva información. Por otra parte, nuestros estudios han comprobado que a estas edades se han alcanzado niveles de desarrollo del pensamiento económico que implican la comprensión de nociones básicas, como la ganancia y el carácter fiduciario del dinero, así como su carácter de medio de intercambio (Denegri, 1995; Denegri, Delval, Ripoll, Palavecinos y Keller, 1998). Así, al evaluar el nivel de Alfabetización Económica en niños chilenos, se concluye que la mayor parte de los escolares de 11 a 12 años se encuentran en un nivel transicional de afianzamiento de su nivel de pensamiento económico, lo que los hace especialmente flexibles y susceptibles a estrategias de intervención que mejoren sus niveles de alfabetización económica (Denegri, Cabezas, Páez, Sanhueza, Vargas, Zapata y Sepúlveda, 2010a).

En cuanto a la comprensión del funcionamiento financiero bancario, los estudios con adolescentes de 14 a 18 años señalan el impacto del estrato socioeconómico, observándose que el nivel de comprensión de las funciones bancarias es más completo en los adolescentes de los estratos socioeconómicos medio y alto, que viven en ciudades multifinancieras. Se aprecia un mayor desconocimiento y un bajo nivel de comprensión de los aspectos evaluados en los sujetos del nivel socioeconómico bajo y residentes en ciudades con funcionamiento financiero limitado o pueblos pequeños, quienes tienden a atribuir, además, al banco una función benefactora, lo que puede estar influido por su escasa experiencia directa con la actividad bancaria y con el predominio de la asistencia 
Estudios Pedagógicos, vol. XL, n. 1, 75-96, 2014

¿CONSUMIDORES O CIUDADANOS? UNA PROPUESTA DE INSERCIÓN DE LA EDUCACIÓN ECONÓMICA Y

FINANCIERA EN LA FORMACIÓN INICIAL DOCENTE

social en el tipo de prestaciones en las que ellos interactúan con el banco. Por ejemplo, acompañar a padres o abuelos a cobrar una pensión (Denegri, Martínez y Etchebarne, 2007).

\subsection{ALFABETIZACIÓN ECONÓMICA EN ESTUDIANTES DE EDUCACIÓN SUPERIOR}

Estudios realizados en estudiantes universitarios (Denegri, Cabezas, Páez, Vargas y Sepúlveda, 2009a; Denegri, González, y Sepúlveda, 2010c) dan cuenta de que sus niveles de alfabetización económica son precarios, por lo cual no manejarían óptimamente conceptos, habilidades, destrezas y actitudes que les permitan comprender efectivamente el mundo, dificultando la toma de decisiones eficientes acordes a sus recursos financieros.

Se ha encontrado también que mientras mayor es la formación económica formal, mayor también es la complejidad y cantidad de información manejada por los sujetos, haciéndose su conceptualización de la economía más abstracta y sofisticada. Sin embargo, el nivel de educación económica formal no aparece sustantivamente asociado a los hábitos de consumo, actitudes hacia el endeudamiento y, en general, a una conducta económica eficiente. Es así como estudiantes que cursaron asignaturas avanzadas en economía, mostraron un desempeño económico en sus finanzas personales tan ineficiente como aquellos sin una educación económica sistemática (Denegri et al., 2006b).

Lo anterior sugiere que la educación económica meramente conceptual, propia de la formación universitaria, sería insuficiente, por sí misma, para modelar hábitos y actitudes responsables frente al consumo. Si bien los universitarios de carreras con formación económica tienen un mejor nivel de comprensión de la economía y también más elementos de juicio para seleccionar vías de consumo más eficientes, ello no basta si no se considera la aplicación cotidiana de estos elementos, el desarrollo de competencias respecto al manejo del dinero y el cambio de actitudes hacia el consumo, los que parecieran instalarse tempranamente como producto de los procesos de socialización económica en la infancia, fundamentalmente en la familia (Denegri et al., 2006b).

En investigaciones con estudiantes de pedagogía, se ha concluido que un profesor que maneje los conceptos y que al mismo tiempo sepa como operar con ellos en lo cotidiano, dispondrá de una valiosa herramienta para transmitir, desde su propia práctica, la información a sus estudiantes de modo simple y efectivo. De este modo estará contribuyendo a la formación de ciudadanos y no consumidores, afianzando las orientaciones de la pedagogía hacia un modelo de educación para participación ciudadana, activa y responsable, como es prevista por el estado dentro de su agenda social (Denegri, Del Valle, Gempp, Etchebarne y González, 2010b).

Como una primera conclusión y considerando los resultados del análisis de brechas, surgen sectores de la población que requieren una intervención en el ámbito de la educación económica y financiera que debería traducirse en acciones focalizadas.

a) Población adulta de sectores socioeconómicos medios. En este segmento surgen dos vertientes de intervención que resultan claves a la luz de los resultados obtenidos en los estudios reseñados.

La primera de ellas debería estar orientada a reforzar y profundizar sus herramientas de comprensión del funcionamiento financiero y económico que les permita tomar decisiones, esto debe necesariamente complementarse con el estímulo del ejercicio reflexivo acerca de los valores y actitudes que están a la base de sus estilos de consumo. El segundo ámbito de intervención debe estar orientado a sus estilos de socialización 
económica e implica por una parte entregar herramientas en relación a las estrategias de socialización que parecen ser más eficientes, por ejemplo, el uso de la mesada. Por otra parte, en este mismo ámbito resulta necesario estimular la reflexión crítica acerca de sus propias concepciones y significados de la paternidad y maternidad vinculadas a la satisfacción de necesidades reales en sus niños.

b) Población adulta de sectores socioeconómicos bajos. En este sector la intervención resulta altamente estratégica por el impacto que ello puede tener en su calidad de vida. Considerando que todas las investigaciones muestran niveles muy insuficientes de alfabetización económica y financiera, es evidente que este segmento requiere de estrategias focalizadas y contextualizadas al tipo de decisiones económicas y financieras que deben tomar, pero que consideren como punto de partida la entrega de conceptos muy básicos, a partir de los cuales se puede ir avanzando en profundidad y gradualidad. Es importante considerar que sus niveles educativos son también más precarios, por lo que los dispositivos didácticos deben estar adecuados a esta realidad.

c) Intervenciones educativas con enfoque de género. Este es un aspecto que conviene considerar dado que los estudios señalan diferencias entre hombres y mujeres, tanto en los niveles de comprensión como en los significados que le otorgan al dinero.

d) Segmento de preadolescentes (tweens) y adolescentes. Dado que las investigaciones señalan que a partir de los 10 u 11 años están presentes tanto las competencias cognitivas como la autonomía relativa, el interés progresivo en el mundo social y una mayor susceptibilidad a la influencia del grupo de pares y los medios de comunicación, este segmento resulta especialmente interesante para focalizar estrategias de educación económica y financiera. El impacto que puede tener una intervención en este grupo etáreo es estratégico, dado que por sus características agrupan en si tres tipos de mercado: un mercado presente por la cantidad de recursos que manejan y su autonomía para tomar decisiones de consumo; un mercado futuro dado que en estas edades se establecen actitudes y patrones de consumo que pueden persistir en la edad juvenil y adulta y un mercado de influencia, por el impacto que tienen en las decisiones de consumo familiar. Por lo tanto, el logro de perfiles de consumo responsable, eficiente e informado puede impactar no sólo a nivel personal, sino también familiar y social.

e) Estudiantes universitarios. Este es un grupo especialmente sensible, que ha sido muy poco abordado en las estrategias de intervención en educación económica y financiera, tanto a nivel del curriculum de formación universitaria, como en esfuerzos desde la institucionalidad pública. Es un segmento que en todas las investigaciones muestra una alta vulnerabilidad frente a la presión del mercado crediticio y comercial, con perfiles de riesgo de endeudamiento y alto consumo impulsivo, a pesar de tener una escasa independencia económica.

f) Profesores y estudiantes de pedagogía. El grupo de los profesores de distintos niveles educativos constituye un segmento estratégico, puesto que dado su rol, constituye una posibilidad de instalación y multiplicación de estrategias educativas orientadas al consumo racional, inteligente y eficiente. No obstante ello, es importante considerar además que poseen un impacto como modeladores de comportamiento, actitudes y valores por lo que estos aspectos deben ser abordados explícitamente como parte de las estrategias educativas. Por otra parte, algunos estudios señalan que es un grupo en el cual sus hábitos de consumo y creciente endeudamiento constituyen factores relevantes de desgaste profesional, incidiendo esto en su salud física y mental, lo que avala la 
necesidad de incluir estos aspectos en los procesos formativos (UNESCO, 2005). Un subgrupo especifico está constituido por los estudiantes de pedagogía, los cuales no han sido considerados como grupo objetivo en las estrategias de capacitación; no obstante ello, es importante incluir estrategias especificas por el impacto que ello puede tener en la conformación de la propia identidad profesional y su futuro quehacer formativo en escuelas y liceos.

\section{UNA PROPUESTA DE CONTENIDOS PARA UN MODELO EDUCATIVO INTEGRADO EN EDUCACIÓN ECONÓMICA Y FINANCIERA}

Como ya hemos señalado, el actual y acelerado proceso de globalización ha dejado huellas en la dinámica económica, social, política, y, especialmente, en los procesos de construcción de identidad social y personal, los cuales cada vez se desarrollan más asociados al consumo (Bauman, 2007; García Canclini, 1995; Lechner, 1998).

Para enfrentar este complejo escenario, se requiere que el individuo construya una visión sistémica del modelo económico social, lo cual implica, por una parte, manejar una serie de informaciones económicas específicas y, por otra, ser capaz de inferir a partir de claves no siempre explícitas el curso de los eventos económicos cotidianos y con ello anticiparse a tomar decisiones que resulten favorables para sí mismo y su entorno (Denegri y Delval, 2002).

A pesar de su importancia, la presencia de la educación económica en los curriculum educativos, tanto latinoamericanos como en chilenos, es precaria. En este último caso, toda la educación económica se desarrolla en sólo 20 a 22 semanas del total de los 12 años de educación obligatoria, apareciendo las temáticas en forma aislada en distintos momentos y en diferentes unidades de la formación, sin existir un eje que las aglutine y careciendo de un modelo pedagógico que considere tanto la génesis de la comprensión de la economía y los procesos cognitivos, afectivos y sociales que la sustentan, ni metodologías que orienten sobre la forma de introducir estos temas en las actividades educativas (Domper, 2004).

En este contexto, resulta necesario definir lo que vamos a entender como Educación Económica, la que se concibe como una acción educativa intencionada, cuyo objetivo es aportar las nociones económicas básicas y las estrategias para tomar decisiones adecuadas que permitan situarse ante la sociedad de consumo como personas conscientes, críticas, responsables y solidarias. Sus componentes se incluyen en el siguiente diagrama (Denegri et al., 2006a). 
Diagrama 1. Componentes de la educación económica

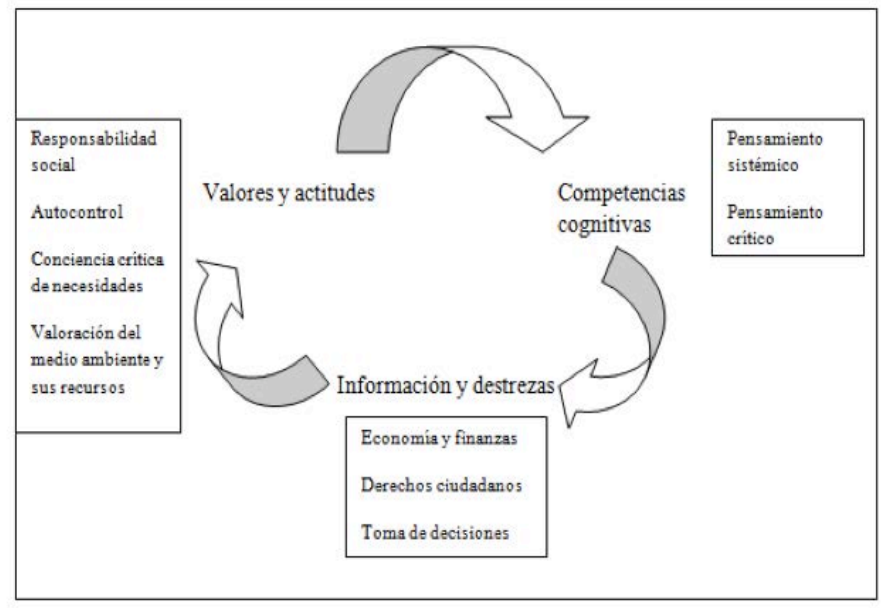

Por su parte, la Organización para la Cooperación y Desarrollo Económico (OCDE) destaca que la Educación Financiera debe ser considerada como un aspecto complementario (no sustituto) de los esfuerzos de regulación y supervisión del sistema financiero, al mismo tiempo que señala su importancia como una herramienta que permite promover el crecimiento económico, la confianza y la estabilidad, en conjunto con la regulación de las instituciones financieras y la protección de los consumidores (OCDE, 2005a, 2005b, 2008, 2009a).

La educación financiera es esencial para ayudar a los consumidores a tomar decisiones bien informadas y beneficiosas que permitan mejorar su situación financiera y fortalecer las eficiencias en el mercado financiero para los consumidores. Los consumidores financieramente capacitados son la primera línea de defensa para el buen funcionamiento de los mercados (Braunstein, 2008).

La evidencia empírica existente demuestra que adultos, tanto de países desarrollados como de economías emergentes que han estado expuestos a procesos de educación financiera, desarrollan comportamientos más proclives hacia el ahorro y la planificación de recursos, entre ellos los orientados a asegurar su previsión (Bernheim, Garrett \& Maki, 2001; Cole, Sampson \& Zia, 2010; Lusardi, 2009). En esta misma línea, se ha encontrado que mayores niveles de alfabetización financiera se relacionan no sólo a la creación de activos, sino también con más capacidades de gestión de la deuda, donde los individuos optan por hipotecas menos costosas, evitando los altos intereses y cargos adicionales (Gerardi, Goette \& Meier, 2010; Lusardi \& Tufano, 2009a, 2009b; Moore, 2003). Esta evidencia sugiere una relación causal directa entre la educación financiera y los cambios en el comportamiento.

Si bien la educación económica y financiera pueden servir para cumplir diferentes propósitos, su efectividad va a depender en gran medida de la claridad, precisión y evaluabilidad de los objetivos que se pretenden alcanzar con ella, el tipo de competencias que se esperan desarrollar en la población y los contenidos y estrategias educativas seleccionadas para su aplicación (Cumbre Latinoamericana sobre Educación Económica y Financiera [NCEE/CEMLA], 2009). 
En cuanto a contenidos, la mayoría de los modelos de educación económica coinciden en que es fundamental incorporar la relación entre al menos tres variables: (1) elecciones económicas, (2) conceptos económicos y (3) metas del comportamiento (Schug, 1982, 1996; Schug \& Hagedorn, 2005).

En el siguiente diagrama se presentan los conceptos articuladores de la educación económica en los cuales coinciden la mayoría de las investigaciones al respecto (Denegri et al, 2006a, 2009b, 2011; Kourlinsky 1996; Laney, 1993; NCEE, 2005).

Diagrama 2. Conceptos articuladores de la educación económica

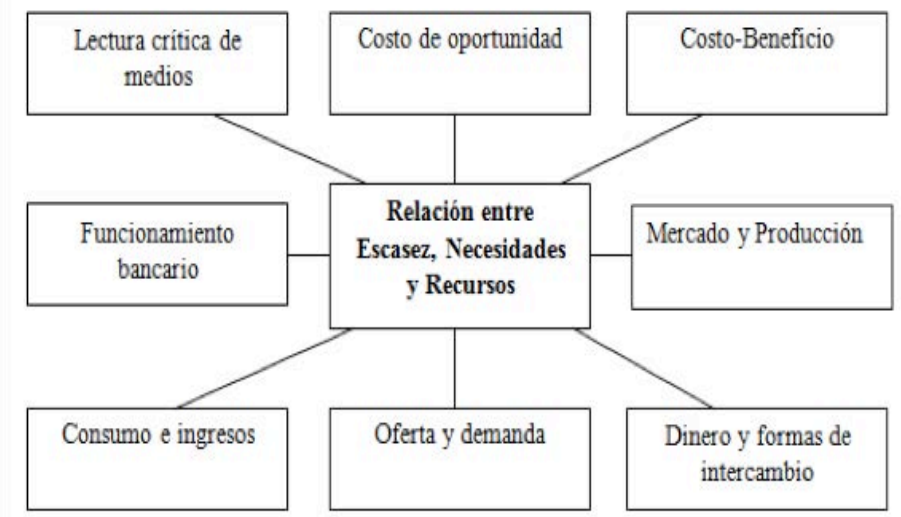

Al respecto, una forma operativa para abordar la articulación de la educación económica y financiera con las políticas educativas está representada en el marco proporcionado por la OCDE, organización internacional a la que pertenece Chile desde el año 2010. En relación a los contenidos específicos que deben abordarse en los programas de educación financiera, el marco de la OCDE sugiere que estos deben clasificarse en contenidos, procesos y contextos. Los contenidos están representados por las áreas de conocimiento; los procesos están constituidos por las estrategias mentales cognitivas de procesamiento de información y toma de decisiones y sus componentes afectivos, actitudinales y valorativos y los contextos están representados por las situaciones en las que se aplican los conocimientos y habilidades.

En el cuadro siguiente, se desglosan los distintos ámbitos que deberían ser abordados en un modelo de educación financiera, a partir de las recomendaciones de la OCDE y de los aspectos que serán incluidos en la prueba PISA 2012² (OCDE, 2009b, 2010, 2011).

1 Cabe señalar que en el caso de Chile, no se incluirá este aspecto evaluativo en la versión PISA del año 2012, pero es evidente que es un ámbito que deberá incluirse en futuras evaluaciones, dados los compromisos del país al estar incluido como uno de los países miembros de la OCDE. 
Cuadro 1. Áreas que deberían ser abordadas en un modelo de educación financiera a partir de las recomendaciones de la OCDE

\begin{tabular}{|c|c|c|}
\hline Contenidos & Procesos & Contextos \\
\hline $\begin{array}{l}\text { a. El dinero y las } \\
\text { transacciones. } \\
\text { b. Planificación y gestión } \\
\text { de las finanzas. } \\
\text { c. Riesgo y recompensa. } \\
\text { d. Mundo financiero. }\end{array}$ & $\begin{array}{c}\text { a. Identificación de } \\
\text { información financiera. } \\
\text { b. Análisis de información } \\
\text { financiera en un contexto. } \\
\text { c. Evaluación de cuestiones } \\
\text { financieras. } \\
\text { d. Aplicación de } \\
\text { conocimientos financieros. }\end{array}$ & $\begin{array}{l}\text { a. Educación y trabajo } \\
\text { (Ingresos, gastos, } \\
\text { préstamos, opciones de } \\
\text { futuro, emprendimiento). } \\
\text { b. Hogar y familia } \\
\text { (Presupuestos familiares } \\
\text { y jerarquización de } \\
\text { necesidades). } \\
\text { c. Individuo (Ingresos, } \\
\text { gastos, cuentas, seguros, } \\
\text { créditos). } \\
\text { d. Sociedad } \\
\text { (Interdependencia, } \\
\text { responsabilidad social, } \\
\text { consumo responsable y } \\
\text { sustentable). }\end{array}$ \\
\hline \multicolumn{3}{|c|}{ Actitudes y valores } \\
\hline \multicolumn{3}{|c|}{$\begin{array}{c}\bullet \text { Tolerancia al riesgo. } \\
\bullet \text { Postergación de impulsos. } \\
\bullet \text { Locus de control interno y autorregulación emocional. } \\
\bullet \text { Actitudes racionales hacia la compra y hacia el endeudamiento. } \\
\text { - Actitudes positivas hacia el Consumo responsable (ecológico o sostenible) y hacia el } \\
\text { comercio justo. }\end{array}$} \\
\hline
\end{tabular}

\subsection{OTRAS COMPETENCIAS NECESARIAS}

\subsubsection{ALFABETIZACIÓN MATEMÁTICA}

Un cierto nivel de aritmética (o alfabetización matemática) es considerado como una condición necesaria para la alfabetización financiera. Habilidades relacionadas con las matemáticas, como el sentido de número, la familiaridad con varias representaciones de números y habilidades en cálculo mental, estimación y la evaluación de la razonabilidad de los resultados son intrínsecos a la cultura financiera. Operacionalmente, la naturaleza de la alfabetización matemática esperada es aritmética básica: las cuatro operaciones (suma, resta, multiplicación y división) con números enteros, decimales y porcentajes comunes.

\subsubsection{COMPRENSIÓN LECTORA.}

Si bien el aprendizaje de la lectoescritura es una condición mínima requerida para la alfabetización financiera, se espera el desarrollo específico de la capacidad de leer e interpretar el lenguaje de documentos financieros. 

EDUCACIÓN ECONÓMICA Y FINANCIERA EN LA FORMACIÓN INICIAL DE PROFESORES

En nuestros estudios con profesores en ejercicio de los subsectores de Comprensión del Medio Social; Lenguaje y Comunicación y Matemáticas, hemos encontrado que este grupo presenta un nivel de alfabetización económica muy precario y ambivalencia entre actitudes racionales e irracionales hacia la pre-compra y la compra. Sin embargo, los profesores reconocen gastar más de lo presupuestado. Respecto al endeudamiento, manifiestan un estilo ambivalente, aceptándolo como una práctica propia del estilo actual de vida que presiona hacia el consumo, pero a la vez manteniendo una actitud de recelo y conservadurismo; además, es interesante discutir acerca de la sensación de no estar cumpliendo adecuadamente su rol como profesores con sus estudiantes, al no asumir en su enseñanza la educación de temas vinculados a la comprensión de la economía (González, Huerta e Inzunza, 2007). En otro estudio complementario con profesores de educación de adultos, se observa la influencia directa del profesor tanto en la comprensión de conceptos económicos como en el modelamiento de pautas de consumo en sus estudiantes (Urrutia, 2007). Estos resultados son especialmente preocupantes si se considera que la evidencia empírica señala que la calidad docente y la capacidad de transferir los conocimientos son un determinante importante en la calidad de la educación económica recibida por los estudiantes, encontrándose una relación positiva entre el nivel de conocimientos económicos del profesor y los niveles de logro de los estudiantes (Allgood \& Walstad, 1999; Becker 2000; Gratton-Lavoie \& Gill; 2009; Walstad \& Soper 1989; Walstad 2001).

Por otra parte, existen estudios que señalan que la falta de formación sistemática en economía durante la formación inicial docente incide en el desarrollo de actitudes negativas de los profesores hacia la enseñanza de estos contenidos cuando ejercen profesionalmente (Becker, 2000; Becker \& Watts, 2001; Kang, Pryor \& Pryor, 2005). Schug, Lopus, Morton, Reinke, Wentworth \& Western (2003) señalan que los profesores de ciencias sociales presentan fuerte resistencia para enseñar contenidos de economía, mostrando una visión de la economía como densa, demasiado abstracta e incluso relacionando su aprendizaje con un aumento del materialismo en sus estudiantes. Concluyen señalando que es probable que las aprehensiones de los profesores frente a la enseñanza de la economía se relacionen con sus procesos previos y experiencias negativas de formación en estos temas durante su educación de pregrado.

De modo complementario, varias investigaciones señalan que un entrenamiento sistemático de profesores y estudiantes de pedagogía que incorpore evaluación del nivel de alfabetización económica, desarrollo de conceptos claves, reflexión sobre sus propios comportamientos económicos y acompañamiento en la implementación de estrategias de educación económica, tiene un importante impacto en los niveles de aprendizaje que alcancen tanto los participantes como sus futuros estudiantes (Denegri, Del Valle, Gempp, Etchebarne y González, 2009b; Kourlisky, 1996; Gratton-Lavoie \& Gill, 2009; Vargha, 2004; Viswanathana \& Venkatesan, 2008; Walstad \& Allgood, 1999; Walstad \& Rebeck, 2001).

Particularmente, Allgood y Walstad (1999) fundamentan la relevancia que tienen los cursos de economía en el desarrollo académico de los docentes, demostrando que aquellos docentes que participan de un programa de educación económica logran un mayor desarrollo en su comprensión, conocimiento y manejo de la economía global y cotidiana, lo que tiene un efecto positivo, tanto en su propia vida financiera como en el aprendizaje de temas económicos en sus estudiantes; de esta forma existe una rentabilidad a largo plazo. 
A partir de estos resultados, nuestra propuesta de un programa sistemático de educación económica para la formación docente inicial incorpora tres aspectos interrelacionandos: 1) evaluación inicial, 2) inmersión teórica y reforzamiento de competencias pedagógicas con seguimiento riguroso y tutoría durante el proceso formativo y 3) evaluación final centrada en la elaboración de un producto tangible por parte de los participantes.

En la etapa de evaluación inicial, debe considerarse la medición del nivel de alfabetización económica y financiera de los participantes así como una caracterización de sus actitudes hacia el endeudamiento y hábitos de consumo para determinar sus perfiles de entrada al programa.

La fase de inmersión teórica y reforzamiento de competencias pedagógicas debe permitir construir, a partir de la reflexión conjunta sobre la práctica, una serie de conceptos comunes que posibiliten crear una estrategia de trabajo coordinado. El proceso formativo debería centrarse en la revisión de los conceptos económicos y financieros articuladores; la apropiación conceptual; la reflexión sobre sus propias posturas frente al tema del consumo y la economía; ejercitación en las diferentes metodologías de enseñanza aprendizaje y las estrategias de planificación de actividades didácticas, incorporando los pasos de experiencia, cuestionamiento y refuerzo mediante la reflexión guiada.

Para ello, conviene reflexionar con los profesores acerca de las características de la etapa de desarrollo de sus alumnos y del creciente papel que juegan los niños y jóvenes como actores económicos. Como soporte pedagógico y a partir de un marco conceptual constructivista, conviene abordar técnicas de elaboración y evaluación de mapas conceptuales, técnicas de trabajo cooperativo y los conceptos básicos de educación económica y financiera que deben ser articulados con los contenidos curriculares afines dentro de los planes y programas educativos vigentes en la educación básica y media. Ello orientado al desarrollo de competencias para la planificación de un proyecto de aula interdisciplinario orientado a la educación económica y financiera.

Durante toda esta etapa, es necesario que los participantes cuenten con una tutoría cercana que permita detectar vacíos de conocimiento y favorezca la apropiación de las estrategias y técnicas didácticas específicas. Un aspecto importante dentro del proceso formativo es la instalación de una actitud de reflexión permanente sobre su propia práctica docente y la coherencia entre las experiencias de aprendizaje de sus alumnos y sus propias experiencias en la toma de decisiones como consumidor. Ello, para aumentar la coherencia actitudinal, considerando que el profesor constituye un modelo de comportamiento para sus estudiantes y que un profesor consumerista (Denegri y Martínez, 2004) tiene mayor probabilidad de fomentar comportamientos de consumo reflexivo.

En el cuadro siguiente se presenta una propuesta general de las distintas temáticas que permitirían abordar la educación económica y financiera en la formación inicial docente.

Cuadro 2. Propuesta general de temáticas para la educación económica y financiera en la formación inicial docente Diagrama 1. Componentes de la Educación Económica

\begin{tabular}{|c|c|}
\hline $\begin{array}{c}\text { Módulo 1: El mundo } \\
\text { del consumo }\end{array}$ & $\begin{array}{c}\bullet \text { Los niños y jóvenes y el consumo: aspectos evolutivos. } \\
\bullet \text { El consumo como telón de fondo de la construcción actual de } \\
\text { identidad. } \\
\text { Autoreconocimiento y reflexión sobre las actitudes personales } \\
\text { hacia el consumo, endeudamiento y ahorro }\end{array}$ \\
\hline
\end{tabular}




\begin{tabular}{|c|c|}
\hline $\begin{array}{l}\text { Modulo 2: } \\
\text { Metodologías } \\
\text { didácticas de soporte } \\
\text { en educación } \\
\text { económica y } \\
\text { financiera }\end{array}$ & $\begin{array}{l}\text { - Las aproximaciones a la educación económica y financiera. } \\
\qquad \text { - Mapas conceptuales. } \\
\text { - Estrategias de trabajo cooperativo. } \\
\text { - La formulación de un proyecto de aula interdisciplinario. } \\
\text { - Evaluación de proyectos de aula interdisciplinarios. } \\
\text { - Desarrollo de destrezas personales y de trabajo en equipo }\end{array}$ \\
\hline $\begin{array}{c}\text { Módulo } 3 . \\
\text { Contenidos } \\
\text { específicos de } \\
\text { educación económica } \\
\text { y financiera }\end{array}$ & $\begin{array}{l}\text { 3.1 Conceptos económicos básicos. } \\
\text { • Escasez y recursos limitados. } \\
\text { • Mercado; producción de bienes y servicios y trabajo. } \\
\text { - Toma de decisiones de consumo y economía personal y } \\
\text { familiar. } \\
\text { • Cómo funciona la banca y los productos financieros. } \\
\text { • La responsabilidad social y ciudadana en el ámbito de la } \\
\text { economía. } \\
\text { 3. 2. Las fuentes de persuasión al consumo. } \\
\text { • Publicidad y medios de comunicación. } \\
\text { • Los mensajes publicitarios. } \\
\text { - La educación para la conciencia crítica de los medios. } \\
\text { 3.3. Las matemáticas y el mundo de la economía. } \\
\text { • Economía: un mundo de números. } \\
\text { • Los números naturales y el dinero. } \\
\text { • Mresupuesto, consumo y ahorro: fracciones y decimales. }\end{array}$ \\
\hline
\end{tabular}

Durante la fase de evaluación, conviene que se oriente a los futuros profesores a la planificación de una unidad didáctica o proyecto de aula, preferentemente interdisciplinario, a partir de las temáticas revisadas y vinculadas con los contenidos curriculares de los distintos subsectores. La elaboración de la unidad didáctica o proyecto debe contar con la asesoría de un tutor, que sirve de mediador y facilitador del proceso de construcción y desarrollo de la propuesta. Transversalmente, se debe trabajar la aceptación mutua; la cooperación para favorecer las buenas relaciones entre colegas; la coordinación de intereses; la tolerancia y la superación de problemas y mantener una reflexión constante sobre los propios estilos de consumo y el modelaje que desde ellos se podrían proyectar hacia sus futuros estudiantes.

Finalmente, es importante la evaluación cualitativa y cuantitativa del resultado del proceso formativo y su impacto en la educación económica y financiera de los futuros profesores y sus familias. Para ello, además de la evaluación de apropiación de contenidos, conviene medir nuevamente el avance en alfabetización económica y financiera y el cambio actitudinal, a la vez que recoger sus percepciones y valoración de los aprendizajes desarrollados. 


\section{REFLEXIONES FINALES}

Las experiencias internacionales parecen indicar que la educación económica y financiera beneficia a los individuos en todas las etapas de la vida. A los niños les hace comprender el valor del dinero y del ahorro; a los jóvenes les prepara para la vida independiente y a los adultos les ayuda a planificar decisiones básicas como la compra de una vivienda, el mantenimiento de una familia, la financiación de los estudios de sus hijos o a preparar la jubilación.

En términos de beneficios globales, en el plano individual la educación económica y financiera, contribuye a mejorar las condiciones de vida de la población al proporcionar las herramientas necesarias para la toma de decisiones relativas a la planeación para el futuro y a la administración de los recursos, así como información pertinente y clara que da lugar a un mayor y mejor uso de los productos y servicios financieros. Así, los usuarios con mayores niveles de educación financiera tienden a ahorrar más, lo que normalmente se traduce en mayores niveles de inversión y crecimiento de la economía en su conjunto.

A su vez, una ciudadanía educada financieramente contribuye a mejorar el desempeño de las instituciones generando un intercambio de información de mayor calidad entre las instituciones financieras y sus clientes; apoyando la construcción y/o restablecimiento de las confianzas en el sistema financiero; aumentando la competitividad e innovación y mayores mecanismos de autorregulación del propio mercado ante la necesidad de rendir cuentas de su acción frente a una ciudadanía informada y activa.

Ya en el año 1973, el Informe Aprender a Ser. La Educación del futuro patrocinado por las Naciones Unidas, señalaba que:

Un aspecto clave para el desarrollo de la democracia, es lograr que cada ciudadano se convierta en gente consciente del desarrollo, así como un consumidor informado, gracias a un conocimiento real de las leyes, mecanismos y piezas de la vida económica de la nación, de la colectividad local, de la empresa, de los conflictos que en ella tienen lugar, de las fuerzas internas y externas que en él actúan, de los medios que se ofrecen a las diferentes clases económicas para influir sobre el reparto del producto social del trabajo, la productividad, la elección de las inversiones y la planificación [...] En la escuela, y por todos los medios extraescolares, la educación económica debe convertirse en uno de los elementos esenciales de la conciencia y de la cultura de las masas (Faure, Herrera, Razzak, Lopes, Petrovski, Rahnema y Champion, 1973: 232).

Se planteaban allí tempranamente una serie de recomendaciones que, en el caso de Chile, constituirían problemas aún no abordados por la agenda educativa y de desarrollo ciudadano. Ello queda en evidencia cuando reflexionamos en torno a las principales brechas señaladas por los resultados de investigaciones, sobre alfabetización económica, donde se constata que los niveles de alfabetización económica y financiera en adultos y adolescentes son rudimentarios, por lo cual no logran desenvolverse en el mundo económico de un modo óptimo ni tomar decisiones eficientes, observándose una brecha general entre los requerimientos de la vida económica y sus capacidades reales de respuesta. En esta misma línea, se constata que el nivel socioeconómico es una variable de alto peso en la comprensión del funcionamiento del mundo económico y financiero, observándose que los sectores más bajos están en una posición de mayor vulnerabilidad frente a la presión del mercado dado que no poseen una alfabetización financiera y económica mínima para ello. 
Si bien, como ya hemos señalado a lo largo de este documento, no se aprecia una presencia sistemática de Educación Económica en el currículo a nivel de Educación Básica y Media, la brecha se hace aún más evidente en Educación Superior. Esta formación sólo está presente en las carreras asociadas directamente al ámbito financiero, donde se aprecia un buen manejo conceptual. Sin embargo, a nivel de sus capacidades de administración de sus propios recursos y toma de decisiones de consumo, se aprecia igualmente una presencia de hábitos impulsivos y de actitudes proclives al endeudamiento; ello implica que no se ha producido un cambio en sus actitudes y valores frente al consumo que les orienten a patrones de mayor eficiencia y responsabilidad su manejo de recursos no siempre resulta ser el óptimo. Dado lo anterior, resulta necesario pensar en intervenciones de educación económica y financiera dirigida a estudiantes universitarios, considerando que son un segmento altamente intervenido por las estrategias de colocación de productos financieros y comerciales.

En esta misma línea, es aún más preocupante que los estudiantes de pedagogía que posteriormente serán los encargados de formar conocimientos, habilidades y conductas económicas a nivel escolar no manejen adecuadamente los contenidos mínimos de economía incorporados en el curriculum, por lo que presume que no lograrán cumplir de un adecuado modo con su rol de educadores.

Es evidente, a partir de estas conclusiones, que se debe insistir en la necesidad de incorporar dentro de los procesos de formación inicial docente el aprendizaje de conceptos económicos claves, integrando al manejo teórico con procesos auto reflexivos que resuelvan sus propias necesidades de interacción cotidiana en lo económico. Ciertamente un profesor que maneje los conceptos y que, al mismo tiempo, sepa como operar con ellos en lo cotidiano, dispondrá de una valiosa herramienta para transmitir, desde su propia práctica, la información a sus estudiantes de modo simple y efectivo. De este modo estará contribuyendo a la formación de ciudadanos y no consumidores, afianzando las orientaciones de la pedagogía hacia un modelo de educación para participación ciudadana, activa y responsable, como es prevista por el estado dentro de su agenda social.

Ello es crítico en un país donde el consumo se ha constituido en el gran articulador de identidad, donde individuos y familias se ven sometidos a un bombardeo constante de estrategias persuasivas complejas y altamente sofisticadas, que incitan hacia el consumo como la salida mágica de los problemas cotidianos y de la búsqueda de la felicidad. Lo cotidiano en nuestro país se caracteriza por la abundancia de artículos y servicios; el culto a los objetos y diversiones y a la cultura mediática, hedonista y materialista. Una sociedad marcada por la lógica del consumo postmoderno, donde todo es efímero; reemplazable y desechable y la búsqueda incesante de lo nuevo, como una especie de talismán para hacer frente a lo que Lipovetsky (2003) llama la era del vacío.

Es en este escenario donde deberán ejercer su función formadora los futuros profesores y resulta poco comprensible que en su formación inicial no se les dote de los contenidos, procedimientos y estructura actitudinal, que les permitan comprender la complejidad de las sociedades de consumo actuales y ejercer una mediación efectiva en sus estudiantes para prepararlos como futuros ciudadanos críticos, reflexivos; responsables socialmente y capaces de ponderar y jerarquizar los eventos económicos y su impacto en sus vidas. 


\section{REFERENCIAS BIBLIOGRÁFICAS}

Allgood, W. \& Walstad, S. (1999). The Longitudinal Effects of Economic Education on Teachers and Their Students. Journal of Economic Education, vol.30, n.2, 99-111.

Bauman, Z. (2007). Vida de consumo. Buenos Aires: Fondo de Cultura Económica.

Becker, W. E. (2000). Teaching economics in the 21st century. Journal of Economic Perspectives, vol.14, 109-119.

Becker, W. E. \& Watts, M. (2001). Teaching economics at the start of the 21st century: Still chalk and talk. American Economic Review, vol.91, 446-452.

Bernheim, D., Garrett, D., \& Maki, D. (2001). Education and saving: The long-term effects of high school financial curriculum mandates. Journal of Public Economics, vol.85, 435-565.

Braunstein, S. (2008). Director Division of Consumer and Community Affairs, Before the Committee on Financial Services, U.S. House of Representatives. Extraído en: http://www.federalreserve. gov/newsevents/testimony/ braunstein20080415a.htm

Cole, S., Sampson, T., \& Zia, B. (2010). Prices or Knowledge? What Drives Demand for Financial Services in Emerging Markets? HBS Working Papers 09-11, forthcoming. The Journal of Finance.

Comisión De Formación Ciudadana. (2004). Informe Final. Chile: Ministerio de Educación.

Commonwealth Bank Foundation [CBF]. (2004a). Australians and Financial Literacy. Sydney: Commonwealth Bank Foundation.

Commonwealth Bank Foundation [CBF]. (2004b). Improving Financial Literacy in Australia: Benefits for the Individual and the Nation, Research Report. Sydney: Commonwealth Bank Foundation.

Cumbre Latinoamericana Sobre Educación Económica y Financiera [NCEE/CEMLA]. (2009). Actas de la Cumbre de Alfabetización Financiera de América Latina, 2009. Washington.

Denegri, M. (1995). El desarrollo de las ideas acerca del origen y la circulación del dinero: Un estudio evolutivo en niños y adolescentes. Colección Tesis en Microficha. Servicio de Publicaciones de la Universidad Autónoma de Madrid. ISBN: 84 - 7477 - 555 - 8.

Denegri, M., Del Valle, C., Etchebarne, S., y González, Y. (2011). Informe Final Proyecto FONDECYT $N^{\circ}$ 1090179. Alfabetización económica y patrones de consumo y endeudamiento en estudiantes de pedagogía: hacia un modelo explicativo. Santiago: Comisión Nacional de Ciencia y Tecnología (CONICYT).

Denegri, M., Cabezas, D., Páez; A., Sanhueza, O., Vargas, M., Zapata, L., y Sepúlveda, J. (2010a) Actitudes hacia el Endeudamiento en Adolescentes de Educación Municipal y ParticularSubvencionada de la ciudad de Temuco. Educación y Humanidades. vol.2, n.1, 46-63.

Denegri, M., Del Valle, C., Gempp, R., Etchebarne, S. y González, Y. (2010b). Alfabetización económica y patrones de consumo y endeudamiento en estudiantes de pedagogía: Hacia un modelo explicativo. Informe avance. Proyecto Fondecyt 1090179. Fondo Nacional de Desarrollo Científico y Tecnológico, Santiago, Chile.

Denegri, M., González, J., y Sepúlveda, J. (2010c). Consumo y Construcción de Identidad en Profesores de Educación Primaria en Chile. EDUCERE - Investigación Arbitrada, vol.49, 345-359.

Denegri, M., Cabezas, D., Páez, A., Vargas, M., y Sepúlveda, J. (2009a). Alfabetización Económica en estudiantes universitarios de la carrera de Psicología. Calidad de la Educación, vol.30, 234-249.

Denegri, M., Del Valle, C., Gempp, R., Etchebarne, S. y González, Y. (2009b). Informe final. Proyecto Fondecyt $\mathrm{n}^{\circ}$ 1060303. Departamento de Psicología. Universidad de La Frontera.

Denegri, M., Lara, M., Córdova, G., y Del Valle, C. (2008). Prácticas de Ahorro y Uso del Dinero en Pre Adolescentes (Tweens) Chilenos. Revista UNIVERSUM, vol.23, n.1, 24-38.

Denegri, M., Martínez, G., y Etchebarne, S. (2007). La comprensión del funcionamiento bancario en adolescentes chilenos: un estudio de psicología económica. Interdisciplinaria, vol.24, n.2, 137-160. 
Denegri, M., Del Valle, C., Gempp, R., y Lara, M. (2006a). Educación Económica en la Escuela: Hacia una Propuesta de Intervención. Estudios Pedagógicos, vol.32, n.2, 103-120.

Denegri, M., Gempp, R., Del Valle, C., Etchebarne, S., y González, Y. (2006b). El aporte de la psicología educacional a las propuestas de educación económica: los temas claves. Revista de Psicología de la Universidad de Chile, vol.15, n.2, 69-84.

Denegri, M., Gempp, R., y Martínez, G. (2005a) Estrategias de Socialización Económica en Familias de Clase Alta y Media-Alta y su impacto en las prácticas de uso del dinero de los hijos. Boletín de Investigación Educacional, vol.20, n.2, 41-60.

Denegri, M., Palavecinos, M., Gempp, R., y Caprile, C. (2005b). Socialização Econômica em Famílias Chilenas de Classe Média: Educando Cidadãos Ou Consumidores? Psicologia \& Sociedade, vol.17, n.2, 88-98.

Denegri, M. y Martínez, G. (2004). ¿Ciudadanos o consumidores? Aportes constructivista a la educación para el consumo. PAIDEIA. Revista de Educación, vol.37, 101-116.

Denegri, M., y Palavecinos, M. (2003). Género y Alfabetización Económica: ¿Oportunidades de Desarrollo o Nuevos Caminos para la Discriminación? Psicología desde el Caribe, Universidad del Norte, vol.12, 76-97.

Denegri, M. y Delval, J. (2002). Concepciones evolutivas acerca de la fabricación del dinero II: Los tipos de respuestas. Investigación en la escuela. Revista de investigación e innovación escolar, $n .48,55-70$.

Denegri, M., Iturra, R., Palavecinos, M. y Ripoll, M. (1999). Consumir para vivir y no vivir para consumir. Temuco: Ediciones Universidad de La Frontera.

Denegri, M., Delval, J., Ripoll, M., Palavecinos, M. y Keller, A. (1998) Desarrollo del pensamiento económico en la infancia y adolescencia. Boletín de Investigación Educacional, vol.13, 291-308.

Domper, M. (2004) ¿Qué aprenden de economía nuestros niños en el colegio? Serie Informe Económico, n.151. Santiago: Instituto Libertad y Desarrollo.

Faure, E., Herrera, F., Razzak Kaddoura, A., Lopes,H., Petrovski, A., Rahnema, M., y Champion, F. (1973). Aprender a ser. La educación del futuro. Madrid: Alianza Editorial, S. A.

García Canclini, N. (1995). Ciudadanía y Consumidores. México: Grijalbo.

Gempp, R., Denegri, M., Caripán, N., Catalán, V., Hermosilla, S. y Caprile, C. (2007). Desarrollo del Test de Alfabetización Económica para Adultos (TAE-A). Revista Interamericana de Psicología, vol. 41, n.3, 275-284.

Gerardi, K., Goette, L., \& Meier, S. (2010). Financial Literacy and Subprime Mortgage Delinquency: Evidence from a Survey Matched to Administrative Data. Federal Reserve Bank of Atlanta.

González, J., Huerta, A. e Inzunza, C. (2007). Estilos de consumo, alfabetización económica y construcción de identidad en profesores de educación básica en la ciudad de Temuco. Tesis para optar al grado de Licenciado en Psicología, Departamento de Psicología, Universidad de La Frontera, Temuco, Chile.

Gratton-Lavoie, Ch. \& Gill, A. (2009). A Study of High School Economic Literacy in Orange County, California. Eastern Economic Journal, vol.35, 433-451.

Herrera, M., Estrada, C., y Denegri, M. (2011). La Alfabetización Económica, Hábitos de Consumo, Actitud hacia el Endeudamiento y su Relación con el Bienestar Psicológico en Funcionarios Públicos de la ciudad de Punta Arenas. Magallania, vol.39, n.1, 83-92.

Kang, R., Pryor, C. R. \& Pryor, B. W. (2005). Using the theory of reasoned action (TORA) to examine K-12 preservice social studies teachers' intentions to teach economics. Artículo presentado en el Annual conference of College and University Faculty Assembly, National Council for Social Studies, Kansas City, MO.

Kourilsky, M. (1996). Generative Teaching and Personality Characteristics of Student Teachers. Teaching and Teacher Education, vol.12, n.4, 355-363.

Laney, J. (1993). Economics for elementary school students: Research -supported principles of teaching and learning that guide classroom practice. Social Studies, 99-103. 
Lechner, N. (1998). The transformations of politics. En Agüero, F. \& Starks, J. (eds.) Fault Lines of Democracy in Post-transition Latin America. Miami: University of Miami, North-South Center Press.

Lipovetsky, G. (2003) La era del vacío: Ensayo sobre el individualismo contemporáneo. Ed. Anagrama

Lusardi, A. (2009). U.S. Household Savings Behavior: The Role of Financial Literacy, Information and Financial Education Programs. En FOOTE, C., GOETTE, L. \& MEIER, L. (Eds.), Policymaking Insights from Behavioral Economics (109-149). Federal Reserve Bank of Boston.

Lusardi, A., \& Tufano, P. (2009a). Debt Literacy, Financial Experiences, and Over indebtedness. NBER Working Paper n. 14808.

Lusardi, A., \& Tufano, P. (2009b). Teach Workers about the Perils of Debt. Harvard Business Review, vol. 87, n.11, 22-24.

Lyons, A. (2004). A profile of financially at-risk college students. The Journal of Consumer Affairs, vol.38, 56-80.

Mcelprang C., Haskell C. \& Jenkins, J. (2005). Financial attitudes and spending habits of university freshmen. Proceedings of the Academy for Economics and Economic Education, vol.8.

Moore, D. (2003). Survey of Financial Literacy in Washington State: Knowledge, Behavior, Attitudes, and Experiences. Social and Economic Sciences Research Center, Washington State University.

National Council On Economic Education [NCEE]. (2005). Survey of the States: Economic and Personal Finance Education in our Nation's Schools in 2004. New York: National Council on Economic Education.

Norvilitis, J. \& Santa María, P. (2002). Credit card debt on college campuses: causes, consequences, and solutions. College Student Journal, vol.36, 356-364.

Norvilitis, J., Sazblicki, J. \& Wilson, S. (2003). Factors Influencing Levels of Credit-Card Debt in College Students, Journal of Applied Social Psychology, vol.33, n.5, 935-947.

OECD. (2011). PISA 2012 Financial Literacy Framework. Extraído de http://www.oecd.org/dataoecd/8/43/46962580.pdf

OECD. (2005a). Improving Financial Literacy: Analysis of Issues and Policies. OECD Publishing..

OECD. (2005b). Recommendation on Principles and Good Practices for Financial Education and Awareness. OECD Publishing, Directorate for Financial and Enterprise Affairs.

OECD. (2008). Improving Financial Education and Awareness on Insurance and Private Pensions. OECD Publishing.

OECD. (2009a). Financial literacy and consumer protection: overlooked aspects of the crisis. Extraído de http://www.financial-education.org/dataoecd/32/3/43138294.pdf

OECD. (2009b). PISA 2006 Technical Report. Paris: OECD Publishing.

OECD. (2010). PISA 2009 Framework: Key Competencies in Reading, Mathematics and Science. Paris: OECD Publishing.

Programa De Las Naciones Unidas Para El Desarrollo [Pnud]/ Instituto Nacional De La Juventud [INJUV]. (2003). Transformaciones Culturales e Identidad Juvenil en Chile. Santiago.

Programa de Las Naciones Unidas para El Desarrollo. [PNUD]. (2002). Nosotros los chilenos: Un desafío cultural. Informe Desarrollo Humano en Chile. Chile: PNUD.

Raven, F. (2005). Financial Literacy: A Basic Skill for Social Mobility [online], Digital Divide Network, 25 May, disponible en: http://www.digitaldividenetwork.org/articles/view. php?ArticleID=420.

Riquelme, L. y Denegri, M. (2008). Alfabetización económica de figuras parentales de diferentes estratos socioeconómicos y género. Documento de trabajo no publicado. Magíster en psicología. Universidad de La Frontera.

Santomero, A. (2003). Knowledge Is Power: The Importance of Economic Education. Business Review. 1-5.

Schug, M. (1982). Economic Education across the Curriculum, Phi Delta Kappa Educational Foundation. 
Schug, M. (1996). Introducing Children to Economic Reasoning: Some Beginning Lesson. The Social Studies, vol.87, n.3, 114-118.

Schug, M. \& Hagedorn, E. (2005) The Money Savvy Pig TM Goes to the Big City: Testing the Effectiveness of an Economics Curriculum four Young Children. The Social Studies, vol.96, n.2, 68-71.

Schug, M. C., Lopus, J. S., Morton, J. S., Reinke, R., Wentworth, D. R. \& Western, R. D. (2003). Is economics your worst nightmare? Social Education, vol.67, 73-78.

UNESCO (2005). Condiciones de trabajo y salud docente. Oficina Regional de Educación de la UNESCO para América Latina y el Caribe. Santiago: OREALC / UNESCO.

Urrutia, J. (2007). Caracterización de los niveles de alfabetización económica en la educación básica de adultos. Tesis de Magíster en Desarrollo Local y Regional. Universidad de La Frontera.

Vargha, L. (2004). Buyer Beware! Economics Activities for Middle School Students. The Social Studies, vol.95, n.1, 27-32.

Viswanathana, M. \& Venkatesan, G. (2008). Understanding and enabling market place literacy in subsistence contexts: The development of a consumer and entrepreneurial literacy educational program in South India. International Journal of Educational Development, vol.28, 300-319.

Walstad, W.B. (2001). Improving Assessment in University Economics, Journal of Economic Education, vol.32, n.3, 281-294.

Walstad, W. \& Rebeck, K. (2001). Teacher and Student Economic Understanding in Transition Economies. Journal of Economic Education, vol.32, n.1, 58-67.

Walstad, W. \& S. Allgood (1999). What does college know about economics? American Economic Review, vol.89, n.2, 350-354.

Walstad, W. B. \& Soper, J.C. (1989). What is high school economics? Journal of Economic Education, vol.20, 53-68.

Yamane, E. (1997). The meaning of "economics education" in Japanese elementary and secondary education: An historical perspective, en Proceedings of the Second Conference of the International Association for Children's Social and Economic Education, Malmö, Sweden: Edge Hill University College/ IACSEE, pp. 101. 